\title{
D'outre-monde : du fantôme de la perte vers une mémoire de l'oubli, ou le moteur caché de la relation à l'autre
}

Départements et Territoires d'Outre-mer. Appellation sans doute révélatrice de la politique française à l'égard de ses anciennes colonies - primat d'un centre sur ses périphéries, dont les usages de la langue ne sauraient être que mésusages - ou alors, littérature. C'est contre pareils postulats que se sont insurgés les auteurs antiet postcoloniaux martiniquais, eux qui n'eurent de cesse de contester une hégémonie culturelle, corollaire de leur propre aliénation, et, avec elle, le cortège d'antinomies qui l'accompagnent: le savant et le populaire, l'oral et l'écrit, le corps et l'esprit, la connaissance et l'esthétique, bref, le même et l'autre. Et cela, ils le firent en français. Mais un français... Non plus la langue de l'autre, mais l'altérité de la langue ellemême, soudain rendue sensible à qui s'en croyait maître et possesseur. Bref, un acte de traduction subversif, où l'épreuve de l'étranger, pour reprendre le beau titre de l'ouvrage d'Antoine Berman (1984), se fait, si inquiétante soit-elle, épreuve de l'étrangeté.

La récente revendication d'une "littérature du tout-monde en français » (2007) s'inscrit dans une telle volonté de déterritorialisation de l'espace, et de la langue. En effet, pour les quelques quarante-quatre écrivains cosignataires du manifeste, plus ne saurait être question d'écrire français, mais bien en français. Dès lors, la francophonie, ce mot singulier qui signe une position d'étrangeté vis-à-vis de la langue et de la culture dont on use, et mésuse, à plaisir, ne saurait plus se concevoir comme allégeance aux normes sociales et linguistiques instituées par un Etat-nation souverain, mais, au contraire, comme ce travail discursif du langage qui, depuis l'écart réflexif ouvert et ouvré sur les lieux mêmes de l'entre-deux des cultures, le motive au-delà de son prêt-à-porter signifiant pour traduire une relation au monde inédite.

Enjeu, sans doute, de toute littérature, ce travail de l'écart du langage n'en revêt pas moins, en situation postcoloniale, une tonalité particulière. Désormais, plus ni centre, ni périphéries, mais, répondant à l'expérience de décentrement qui fait l'obligatoire de la condition du sujet postcolonial, des frontières en tous lieux fracturées d'ailleurs, et qui ne sauraient plus se faire les garantes d'aucune identité territoriale. Mieux encore: de découvrir l'adéquation présumée des deux notions d'identité et de territoire comme l'effet d'un dispositif de pouvoir - donc, au sens foucaldien, de contrôle du corps - qui masque dans le procès de son invisibilisation le processus de 
territorialisation identitaire en quoi il consiste effectivement, et l'aliénation à laquelle il confine.

La logique de la conquête, qui trouve son prolongement dans la politique culturelle assimilationniste française - le dogme, pérenne, de l'intégration, et son prix impossible, l'éradication de toute différence sensible -, laisse en effet le sujet postcolonial seul aux prises avec une différence qui lui est sans cesse réitérée dans le temps même où elle lui est déniée. Un «double-bind» déjà bien analysé dans Peau noire, masques blancs par le psychiatre martiniquais Frantz Fanon, et qui s'origine, non pas tant dans le traumatisme de l'esclavage, que, singulièrement, dans la scène de l'abolition elle-même. Hypocrite invitation à l'identité qui n'offre à l'autre voie d'expression qu'à la condition folle d'embrasser la logique qui l'a réduit en esclavage, et ce jusqu'à en dénier l'épreuve même, son impossibilité est la condition de son octroi. C'est, en effet, dans la mesure même où le sujet postcolonial ne peut se soumettre à une telle induction normative qu'il tombe tout entier sous son joug, frappé d'altérité là même où le sentiment narcissique d'identité - à soi - de l'ancien colon s'en voit, en retour, conforté d'autant. Singularité d'un tel dispositif de pouvoir : réussir, en échouant.

Mais pour être résolument «moins que un et double», comme l'indique Homi Bhabha, toujours tout à la fois en défaut, et en excès, vis-à-vis du modèle idéalisé, et intériorisé comme idéal, du «sujet universel», le sujet postcolonial tient, précisément, sa force subversive de cette impossible adéquation à la norme qui lui offre d'en réfléchir, et les cadres et la dynamique. Mélancolie non seulement spatialisante, mais temporalisante, elle rend en effet patent le tour extraordinairement ironique de la logique sociale qui, à tenir toute marque d'altérité pour symptôme d'aliénation, ne parvient jamais, là même où elle cherche à lui échapper, qu'à la produire effectivement; et la renverse. Car si un tel dispositif de pouvoir réussit, paradoxalement, en échouant, il n'en secrète pas moins le germe de son possible retournement : celui, tout aussi paradoxal, d'un échec réussi du désir narcissique qui, loin de mettre terme à la mélancolie du sujet postcolonial, l'érige en véritable aporétique, et poétique, de la relation à l'autre.

Le néologisme de « créolisation », forgé par Edouard Glissant, donne à cette traversée par l'autre son tour mélioratif, là où il n'existait jusque là en français d'autre mot pour la signifier que ceux, péjorativement connotés, d'altération et d'aliénation'1. Revers de la violence de la rencontre, ce processus créatif, et jamais achevé, dans et par lequel s'élaborent en permanence des modalités de relation inédites entre les cultures. Aussi bien, ce "mode de l'emmêlement» (Glissant Poétique 103) qu'est, pour le philosophe martiniquais, la créolisation, offre au décentrement son tour

${ }^{1}$ Il est notable que le texte développant la notion anthropologique de «transculturation », née à Cuba sous la plume de Fernando Ortiz, n'ait toujours pas été traduit en français... 
désirable : celui d'une relance sans fin du désir dans l'opacité du monde, où l'altérité elle-même devient objet de désir - et ce, dans la mesure même où elle est, irrémédiablement, hors d'atteinte.

Alternative à l'aliénation coloniale, la poétique de la Relation se veut aussi alternative à la "mélancolisation des rapports sociaux », pour reprendre l'expression d'Alain Ehrenberg, à laquelle nous affronte le précipité de notre actuelle mondialisation. À l'heure, en effet, où, sous le coup du choc des cultures, les normes que nous pouvions encore, jusque là, tenir pour inaliénables, implosent, troublant irrémédiablement les frontières établies du même et de l'autre comme du dedans et du dehors, et n'offrant en retour que l'image, effrayante, de l'imprévisible, le « chaosmonde ", toujours selon l'écrivain martiniquais, serait désormais à envisager à l'image, fractale, d'un archipel antillais confronté, depuis l'expérience du transbordement de l'Afrique vers les Amériques, au décloisonnement radical des frontières identitaires et territoriales - sous les auspices, donc, de la créolisation qui en signe la possible élaboration culturelle.

Un nouvel imaginaire dont l'espacement au monde prôné par le philosophe viendrait ainsi contrecarrer la menace double d'une globalisation états-unienne dont le visage désormais amène de la ségrégation, sous les auspices du multiculturalisme, n'est jamais, sous couvert de «respect» des différences, que le masque de leur allégeance assermentée aux valeurs de la constitution qui les transcende, et les annule; de leur exacerbation réactive, sous le mode défensif d'un repli identitaire qui, faute de pouvoir jamais en revenir jamais à l'âge d'or tant vanté, a tôt fait de se « rétroprojeter » en agression terroriste.

Dès lors, l'ouvrage francophone d'une langue française devenue, à la faveur de ce déplacement d'échelle, langue mineure parmi les langues mineures, figure l'un des lieux possibles de résistance, et à l'hégémonie anglo-américaine, et à une francité jalouse de ses prérogatives - et ce, dans la mesure même où cet ouvrage est, aussi, ouverture : loin de vouloir en figer l'essence, et d'en faire ainsi, au propre comme au figuré, une langue morte, il la maintient au contraire vive, toujours disponible au frottement d'autres langues, et d'autres cultures, qu'elle avive avec elle - à l'instar de l'œuvre du poète québécois Gaston Miron, qui trouve ainsi place dans les lignes du Traité du tout-monde du théoricien de la créolisation (Glissant Tout monde 224).

La poétique de la Relation à laquelle nous convie Glissant nous invite ainsi à nous déprendre de l'obsession identitaire dont la projection spatiale, et ce qu'elle soit universaliste ou différentialiste, ne confine jamais, entre ingérence et terrorisme, qu'à une paranoïa tout aussi meurtrière que mortifère, lors même que l'expérience assumée du décentrement, elle, n'a de cesse de se faire moteur de créativité sociale et culturelle. Dès lors, l'espace (y compris linguistique) se prend au jeu du temps, et, 
terre mais non plus territoire, est ainsi rendu à sa pleine historicité, qui ne saurait plus se réduire à l'histoire de son annexion, ni même de ses annexions successives, mais redéployer, au contraire, dans la tessiture des multiples facettes qui en trament l'infini feuilletage, celle de sa créolisation. Comme le veulent les coauteurs des deux manifestes rédigés, pour le premier, à l'encontre de la formation d'un ministère de l'identité nationale en France, et, pour le second, en adresse à Barack Obama (Chamoiseau et Glissant Obama), les murs doivent tomber - et, si frontières il y a, celles-ci ne doivent valoir que de distinguer, non plus pour séparer, mais pour relier (Chamoiseau et Glissant Les murs 18-19).

Mais n'est-ce pas là nouvelle utopie qui, à vouloir ériger la créolisation en figure paradigmatique d'un possible nouveau "Nouveau Monde", se voue à méconnaître, pour mieux l'annihiler, la vie sociale et psychique du pouvoir qui en traverse l'ordre et les désordres - et ce, jusqu'à en devenir aveugle à l'effet de pouvoir qu'elle est, elle-même, susceptible d'induire ? La poétique mondialisée du décentrement génère, en effet, un nouvel implicite du discours qui, pourtant, ne l'organise pas moins dans son entier : à l'instar de la métaphore deleuzienne dont se réclame le philosophe martiniquais, l'identité-rhizome, la créole, ne prend sens que par opposition à l'identité-racine. - Et comment, en effet, penser le Multiple, si ce n'est par contraste avec l'Un ? Comment le décentrement, sans l'idée de centre ? Or, le tribut payé au modèle auquel elle s'adosse pour le renverser est, sans doute, plus conséquent qu'elle ne le voudrait - et ce, dans la mesure même où elle repose sa projection (sur l'autre), corollaire de son déni (en soi).

Car si «tout dominant est secrètement conquis", si, "en prenant le monde, l'Occident s'est aussi fait prendre par lui », si « la force brutale et aveugle livre celui qui l'exerce à d'imparables faiblesses », bref, si l'identité-racine, à vouloir conquérir le monde, se voue à sa propre perte, inéluctablement prise au piège de sa propre altération (à entendre ici sans connotation, ni péjorative, ni méliorative) au contact d'autres langues, et d'autres cultures ; si, en retour, " les vaincus du marché-monde [...] ont reçu en ajoutement les ombres et les merveilles des vainqueurs » (Chamoiseau, Glissant 2007 : 14), rien ne dit que ce métissage soit créolisation, rien ne garantit que ce multiple soit le Multiple prophétisé, et, bien que Glissant appelle la Relation (Poétique 211) à perdre sa majuscule pour se reverser dans le monde, pour l'heure tout autant fétichisé que l'Un décrié.

Les Antilles, en effet, ne sont jamais créoles qu'en vertu de l'histoire qui les y a astreintes - et cette histoire est coloniale. Si celles-ci, comme l'entend Roger Toumson (1998), sont donc bien vouées au désillusionnement identitaire, celui-ci n'a pourtant, a priori, rien d'heureux. Au contraire, ce qui, symptomatiquement, ne cesse d'y être joué, c'est, à travers la quête impossible du «vrai » nom, celui qui n'aurait pas été recouvert par les oripeaux du colonialisme, la souffrance que signifie sa perte, et qui 
signe tout à la fois le caractère utopique, et de la logique identitaire, et de la créolisation. Pour l'une comme pour l'autre, en effet, tout se passe comme si rien n'avait jamais été perdu, ni jamais été désiré (re)trouver. Un déni qui se nourrit, paradoxalement, de ce qu'il refoule : qu'il s'agisse d'en revenir à un âge d'or qui lui préexisterait, ou de prophétiser l'avènement d'une ère nouvelle qui la consacrerait, $c^{\prime}$ est toujours fonction de l'implicite de cette perte que s'organise le discours dans son entier, ainsi devenue moteur inconscient du tout aussi inconscient désir d'en annuler le drame.

Guère étonnant, dès lors, que l'«identité-racine» n'ait de cesse de revenir, symptomatiquement, dans le discours qui entend s'en défaire. Fantôme du discours, elle le hante dans la mesure même où elle lui est indispensable, hantise nécessaire à son rejet, figure de repoussoir sans cesse ramenée à la vie dans la tentative toujours vaine de sa mise à mort - exactement à l'instar de l'altérité dans le discours identitaire. Cette ambivalence qui fait, selon Judith Butler, la marque symptomatique caractéristique de l'affection mélancolique, dans laquelle l'objet répudié du désir, conservé dans la psyché en tant que pouvoir de renoncement, se découvre ainsi, paradoxalement, comme sa source d'existence la plus précieuse, trace, en effet, et dans un cas comme dans l'autre, les sillons d'un deuil impossible.

Or, si, comme elle l'entend dans La vie psychique du pouvoir, de la relation à l'autre, nous ne pourrons jamais faire le deuil, révélant ainsi l'écueil du dispositif de pouvoir identitaire - lui qui voue ses sujets à reconduire, obsessionnellement, le déni de l'altérité qui les traverse, lors même que celle-ci est, pourtant, la condition même de son intériorisation (l'identification est toujours, d'abord, identification à un autre) -, il semble que nous ne puissions pas d'avantage faire le deuil du désir de l'annihiler, et d'abord dans la mesure où sa rencontre est toujours, jusque dans l'expérience du miroir où la situe Lacan, le lieu d'un effroi - effroi et désir dont le commun déni a, précisément, débouché sur l'utopie coloniale.

S'il est, en effet, une utopie francophone, c'est d'abord, comme le rappelle Roger Toumson (1998), la coloniale - celle du « Nouveau Monde ». Parfaite chimère que l'on pourrait modeler à sa guise, la fabrique de ce paradis tant vanté par les premiers littérateurs créoles - blancs, s'entend - ne s'avère autre, sorti des ornières propagandistes des esclavagistes, qu'une entreprise psychotique de «déréalisation du monde ». Les mots sont de Fanon (1961), à qui nous devons d'avoir déconstruit la mécanique folle de l'aliénation coloniale : celle d'un gigantesque déni de réalité où l'utopie fantasmée se fait fantasme d'omnipotence et qui, à vouloir occuper la place $\mathrm{du}$ réel, l'assassine bien plutôt. Perdue au moment même où elle frotte la réalité, comme le veut le titre de l'ouvrage de Roger Toumson, l'utopie vire en son contraire - ou plutôt se donne pour ce qu'elle est : une échappée du monde qui ne saurait exister, comme le veut l'étymologie, en aucun lieu - sinon pour le nier. 
Aussi bien toute l'histoire antillaise est-elle toujours déjà le lieu, non d'un retour, mais d'une revenance. Le retour, quant à lui, est impossible : toute origine perdue ; toute identité interdite. La Négritude de Césaire (qui ne fut ni tout à fait celle de Senghor, ni tout à fait celle de Damas) ne dit pas autre chose : non pas tant la filiation à l'Afrique, que la filiation rompue à l'Afrique - où se noue, précisément, le drame colonial.

« À qui fera-t-on croire que tous les hommes, je dis tous, sans privilège, sans particulière exonération, ont connu la déportation, la traite, le collectif ravalement à la bête, le total outrage, la vaste insulte, que tous, ils ont reçu, plaqué sur le corps, au visage, l'omni-niant crachat? Nous seuls, Madame, vous m'entendez, nous seuls, les nègres! Alors au fond de la fosse ! C'est bien ainsi que je l'entends. Au plus bas de la fosse. C'est là que nous crions; de là que nous aspirons à l'air, à la lumière, au soleil. » (Césaire La tragédie 59)

Or, c'est bien à l'insulte que Césaire met une majuscule (Nègre, et non pas Noir), elle qu'il choisit d'assumer dans sa chair pour mieux réfléchir l'ombre portée des « civilités » coloniales elles-mêmes - à la manière d'une éclipse. Mieux que cela encore : en prenant ainsi le parti délibéré d'incarner le motif aveugle de sa propre déshumanisation, en la rendant, donc, incontournable, il interdit à quiconque de faire, une nouvelle fois, fi de sa propre intime et inquiétante étrangeté. Car la tragédie, cette fois, de la décolonisation, se joue bien là. Dans la volonté sourde, et toujours vaine, d'annuler le drame, de nier la négation elle-même, pour l'enterrer au plus bas de la fosse où nul ne se serait jamais trouvé. Nouveau déni, non de réalité, mais de cette altérité radicale qui la contient et l'excède, et qui n'est pas tant la mort que le processus de déréalisation lui-même - où la mort elle-même perd sens.

Et si la critique vaut à l'encontre des anciennes puissances coloniales, elle s'adresse au moins autant à ceux qui, pour en avoir subi le joug, se prennent désormais d'euxmêmes au piège de l'aliénation induite. Rêve-t-on de répondre à l'hypocrite invitation à l'identité de l'ancien colon jusqu'à tenter, compulsivement, de se désincarner dans la toute mythique transparence du sujet universel (puisque la blancheur, comme le rappelle Homi Bhabha, ne vaut pas tant comme couleur, que comme absence de couleur) ? Il n'y a là rien d'autre, comme le veut l'ouvrage de Frantz Fanon, que des «masques blancs», sur une «peau noire ». Grinçant simulacre, et tragique ironie où, à vouloir effacer la trace obscure de la chair, celle-ci n'a de cesse, à chaque nouveau coup porté, de crier, symptomatiquement, sa réalité blessée. Fantasme-t-on alors, par choc en retour, d'une Africa mater qui, Vierge noire, redeviendrait le souverain giron qu'elle n'aurait jamais cessé d'être ? Elle est déjà violée, et ce viol est inscrit dans la chair métisse autant que dans les rouages de la société, et de la psyché, (post)coloniales.

1848 : Abolition de l'esclavage. "Reconnaissance sans lutte », dit Fanon (Peau noire 176). En d'autres termes, une scène de révolte avortée, et détournée à son 
propre profit par le pouvoir colonial, qui substitue ainsi au désir et à la colère des anciens esclaves ses seules valeurs, sans que leur bien-fondé puisse être encore contesté. "Le Nègre est un esclave à qui on a permis d'adopter une attitude de maître. Le Blanc est un maître qui a permis à ses esclaves de manger à sa table » (Peau noire 178). Peuple d'affranchis plutôt que d'hommes libres, les anciens esclaves furent ainsi privés, non seulement de la possibilité de se différencier de la logique sociale qui les avait asservis, mais du moment même de l'interrogation réflexive qui aurait pu les conduire à l'élaboration de modalités d'existence sociale autres.

Perte non reconnue comme telle, et comme telle toujours en défaut de signification dans l'espace social, elle est dès lors vouée à ne trouver, indicible qui organise le champ du dicible, que voie d'expression symptomatique. Aliénation au tour mélancolique, elle laisse les sujets (post)coloniaux seuls aux prises avec le sentiment d'une souffrance sans nom. Une souffrance au futur antérieur, en quelque sorte, où, faute d'avoir encore les mots pour le dire, on ne sait pas de quoi l'on souffre. Et, puisque l'on ne saurait dire de quoi il retourne, c'est donc qu'il (n')y a rien.

Effet d'un dispositif de pouvoir qui s'invisibilise comme tel dans le procès de sa négation, et qui, ce faisant, la redouble (la double négation semble en effet faire la marque symptomatique caractéristique du déni de réalité de la perte impulsé par un appareil normatif), cette souffrance sociale disparaît ainsi comme telle pour ne plus sembler qu'émaner des sujets (post)coloniaux eux-mêmes. S'il n'y a rien, en effet, aucune raison de ne pas être normal. Commode manière de réitérer à l'autre sa différence tout en prétendant la lui dénier, et de s'assurer ainsi de sa propre indifférence... narcissique.

Rouage pervers, que celui de ce "narcissisme obscène ", ainsi que le qualifie Fanon (1961), où l'ancien colon, pour mieux s'assurer de sa propre complétude identitaire, en vient à aliéner son autre dès lors en proie à un désir ambivalent : tout à fois être nié dans son identité (à l'autre), et nier cette négation identitaire. On ne se surprendra plus, dès lors, des lignes singulières que le psychiatre chapitre à la question de la reconnaissance de l'homme noir :

«L'ancien esclave exige qu' on lui conteste son humanité. Quand il arrive au nègre de regarder le Blanc farouchement, ce dernier lui dit: "Mon frère, il n'y a pas de différence entre nous. » Pourtant le nègre sait qu'il y a une différence. Il la souhaite. Il voudrait que le Blanc lui dise tout à coup : «Sale nègre. » Alors, il aurait cette unique chance - de «leur montrer... » Mais le plus souvent il n'y a rien, rien que l'indifférence, ou la curiosité paternaliste. » (Peau noire 179)

Or, en situant d'abord l'action, non dans la prise d'indépendance haïtienne, mais, audelà, au moment de l'indépendance elle-même, puis en effectuant la réalisation, dans la temporalité de la pièce, du mouvement contrarié d'une téléologie noire, c'est précisément à cette perte double - perte du temps de l'interrogation réflexive, perte de la conscience de la perte elle-même - que Césaire offre son issue symbolique. 
«On nous vola nos noms!» Grondant par la bouche de Douta Seck, c'est Christophe, qui parle, l'esclave devenu roi d'Haïti, rendant ainsi signifiante la perte d'image symbolique à laquelle s'affronte tout un peuple, prix inavoué de l'arrogance narcissique de qui, à vouloir usurper la place du neutre universel, l'hystérisa à sa démesure. "Sentez-vous la douleur d'un homme de ne savoir pas de quel nom il s'appelle ? A quoi son nom l'appelle ? » (37) Le drame colonial ainsi posé, commence la tragédie de la décolonisation, où l'épopée de la prise d'indépendance se meurt.

«De noms de gloire je veux couvrir vos noms d'esclaves » (37)! Elan fabuleux d'un homme qui arracha le cours de l'histoire à son lit pour ériger à la face du monde la Citadelle qui rendrait sa dignité à son peuple et qui, pourtant, donna pour prix de sa liberté reconquise un nouvel ordre tyrannique, étrangement modelé sur celui qu'il s'agissait de renverser. "Ce roi noir, un conte bleu, n'est-ce pas ? Ce royaume noir, cette cour, parfaite réplique en noir de ce que la vieille Europe a fait de mieux en matière de cour ! », comme le relève, non sans malice, le baron Vastey (31). Et, plus loin, plus grave: «L'Histoire pour passer n'a parfois qu'une voie, et tous l'empruntent. - Si bien, constate en retour l'une des dames de la Cour, que celle de la liberté et de l'esclavage se confondraient. »(80)

Et, donnant à cette souffrance sans nom celui, crevé d'infortune, d'une Afrique nègre au moment même où Christophe entreprend d'en rémunérer le défaut à coups de titres de noblesse - tour paradoxal d'un premier acte "bouffon et parodique » où « le sérieux et le tragique se font brusquement jour, comme le veut la didascalie inaugurale, par déchirures d'éclairs » (18) -, de rendre ainsi signifiant le cercle vicié auquel se prend au piège, à l'instar de l'ancien colon, le sujet postcolonial, où le sentiment mélancolique d'une insuffisance identitaire se fait le moteur d'une course folle, et toujours vaine, à sa propre indifférence narcissique - mais sans plus pouvoir, quant à lui, se solder dans aucune projection.

Car à l'heure où l'homme se retrouve seul aux prises avec lui-même, il lui est dès lors impossible de se décharger sur l'autre du poids de sa souffrance, et d'éluder ainsi ses propres contradictions - sauf à les rendre plus criantes encore - « la liberté par les moyens de la servitude » (80) -, et à provoquer ainsi son propre suicide social, que signe le suicide du roi.

Tragique ironie, où le héros, à tenter d'échapper à son destin maudit, s'y précipiterait tout entier, signant ainsi la fatalité résignée d'une "nouvelle naissance » (37) impossible ? Certainement pas. Pas de héros, chez Césaire, et moins encore de deus ex machina pour en condamner la démesure. 
Tout l'enjeu de la pièce tient, au contraire, à montrer l'humanité de la démarche de Christophe. Et, nous invitant ainsi à traverser le miroir, de nous amener à comprendre, au-delà de l'apparence bouffonne de la parodie de Cour que l'ancien esclave s'ingénie à modeler sur celle de son ancien maître, et à travers la dictature qu'il établit pour mieux forcer le destin, le désir de grandeur de l'homme qui voulut, de la fosse à la Citadelle, donner à son peuple figure. Qui, dès lors, pour l'accuser de vouloir outrepasser les bornes mesurées du temps - lui qui, pour avoir été hier dépouillé de toute existence symbolique, entend l'arracher à demain? Alors, à Madame qui, depuis "sa petite tête crépue ", l'accuse de trahir sa charge, et d'en demander trop, oublieux qu'il fut lui-même esclave, à son peuple, Christophe tonne : «Je demande trop aux hommes! Mais pas assez aux Nègres, Madame! (59) Car c'est précisément en vertu de ce déni d'humanité qu'il leur en faut faire plus, et mieux, que tout autre - ou l'inertie veule et complaisante, ou le risque assumé, si incertaine en soit l'issue, de la bataille livrée.

«Peuple haïtien, Haïti à moins à craindre des Français que d'elle-même! L'ennemi de ce peuple, c'est son indolence, son effronterie, sa haine de la discipline, l'esprit de jouissance et de torpeur. Messieurs, pour l'honneur et la survie de ce pays, je ne veux pas qu'il puisse jamais être dit, jamais être soupçonné dans le monde que dix ans de liberté nègre, dix ans de laisseraller et de démission nègre suffiront pour que soit dilapidé le trésor que le martyr de notre peuple a amassé en cent ans de labeur et de coups de fouet. » (La tragédie 29)

Bref : « la liberté, sans doute, mais pas la liberté facile ! »(22).

Et que l'on ne s'y trompe pas: point n'est ici question de mimétisme forcené de l'ancien maître, mais bien de s'en arroger les pouvoirs pour subvertir l'ordre social lui-même. Sans doute, affronté à l'épineuse question de la fondation de l'Etat, y cherche-t-il dans un premier temps modèle - mais pour mieux l'infléchir : si royaume il y a, celui-ci est bel et bien noir. Car Christophe ne veut pas seulement se faire reconnaître à la hauteur de l'ancienne puissance coloniale : c'est sa différence qu'il entend ériger à la face du monde. À qui ne voudrait voir dans le Royaume d'Haïti que caricature de l'ancien ordre colonial, le rappel de cette phrase silex : «Il est temps de mettre à la raison ces nègres qui croient que la Révolution ça consiste à prendre la place des Blancs et continuer, en lieu et place, je veux dire sur le dos des nègres, à faire le Blanc. » (84) - D'aucuns, parmi les chefs d'Etat des nouvelles indépendances africaines qui constituaient l'aréopage du Premier Festival Mondial des Arts Nègres de Dakar, en 1976, de se lever, et de quitter la salle.

Exigence lucide et forcenée de Christophe, et d'abord vis-à-vis de lui-même, mais qui le coupe peu à peu d'un peuple qui, lui, n'est pas prêt à sacrifier sa vie sur l'autel de sa propre édification. Au moment où il entend la faire advenir envers et contre tous, commence la tyrannie, où la liberté affichée se retourne en servitude effective. Pris au piège des rêves de grandeur auxquels il asservît son peuple, lui qui emporta d'abord l'enthousiasme des foules, de ne parvenir qu'à susciter révolte et trahison - pour en 
payer le tribut. Le roi se suicide et emporte dans la tombe le noble idéal qu'il portait par devers son peuple - et par devers lui.

Troublante pièce qui, pour accompagner la tragédie d'un homme ivre de grandeur et de mélancolie, ne s'en fait jamais ni l'exaltation, ni la caricature burlesque, mais s'attache bien plutôt à nous le rendre étrangement familier.

"On me demande souvent: “Etes-vous Christophe oui ou non ?" La réponse n'est pas simple. Je suis choqué par toute une série d'attitudes du Roi Christophe, qui a un côté "nouveau riche », un côté "Monsieur Jourdain ». Et puis, par les moyens extrêmement brutaux, le côté « despote » du personnage qui ne peut avoir mon approbation. Mais le Roi Christophe n'est pas un héros, c'est un homme, dans toute sa complexité, et c'est cela qui est dramatique, pathétique. L'originalité de ma pièce, c'est de montrer l'aspect multiple des gens. » (Césaire et Chraibi 1965)

Et c'est précisément en cela que Césaire offre son issue à l'aliénation coloniale : en clivant la différence entre soi et l'autre pour mettre en perspective la logique de pouvoir qui nous travaille d'ordinaire, bon gré mal gré, à notre insu.

Passage parmi les plus significatifs du Cahier pour un retour au pays natal : celui du nègre dans le tramway. Là, confronté à l'image moquée de ce nègre dégingandé aux jambes par trop grandes et à la moue par trop lippue, Césaire cède, policé parmi les policés, à l'inertie railleuse - pour sûr, « il était COMIQUE ET LAID. » (Cahier 38) Point question ici de parjure difficile ou de trahison douloureuse, non, juste le confort de l'abandon. "Lâcheté », et « lâcheté retrouvée » (38), qui signe le désir sourd de négation de cet autre auquel on refuse, au point de le défigurer, d'avoir jamais fait figure d'alter ego. Aveu troublant, sans doute, chez qui ne voudrait voir en Césaire que l'héroïque Rebelle de sa première pièce, Et les chiens se taisaient - lui qui défia, et à mort, l'architecte aux yeux bleus qui présida aux destinées coloniales. Aveu troublant, donc, que celui de cette méconnaissance complice qui, depuis les ombres de la psyché, appelle au meurtre de sa propre chair. Or, tout le courage tient dans la lucidité de l'aveu.

De la bouche de Césaire, elle rend, précisément, criant, combien le désir veule de se complaire dans la jouissance d'une identité pleine et indifférenciée passe par le déni et la projection de sa propre altérité sur cet autre qui, pour menacer d'en fragmenter l'unité, s'en retrouve, dès lors, lui-même morcelé. Le nègre démantibulé de Fanon (1952), celui dont le schéma corporel, attaqué de toute part, s'effondre, ne l'est pas par le regard de l'autre, mais par son absence. Regard aveugle, tout entier accaparé à aliéner son inquiétant reflet pour mieux s'abîmer dans la contemplation de sa propre image idéalisée, il ne réfléchit rien, ni personne - les revenants, c'est bien connu, n'ont pas de reflet.

Or, si les revenants n'ont pas de reflet, l'effroi naît, précisément, de ce que personne se tienne cependant là, absence tangible, trou noir qui absorbe la lumière plutôt que de 
la réfracter, et abîme dans la nuit aveugle le regard médusé de qui, faute de pouvoir encore s'en détourner, reste un instant figé dans la quête impossible de son reflet soudain, à son tour, évanoui. Force de la Négritude de Césaire : déchirer le voile entre soi et l'autre, et procéder au meurtre symbolique de l'utopie identitaire - plutôt qu'à celui des hommes qui, quelle que soit leur couleur, s'efforcent, et toujours en vain, d'en gagner l'éternité figée.

Car si Christophe rêve d'une «nouvelle naissance » qui ferait feu de tous les oripeaux de l'ancien ordre colonial, il n'en reste pas moins fasciné par sa puissance, ses efforts tout entiers tendus à faire reconnaître son propre idéal normatif à la hauteur de celui de l'ancien maître, depuis le faste dont il s'entoure à cet effet, à l'érection de la Citadelle à laquelle il épuise le corps social. Et, affronté à l'image fantasmatique de la toute-puissance de l'ancien colon, de rester en proie à l'illusion coloniale qui le conduit, à vouloir à tous coûts donner ses lettres de noblesse à son peuple, à prendre sa démesure elle-même pour destin: celle d'une Histoire victorieuse - elle qui n'a qu'une voie - dont il ne brise le cours ${ }^{2}$ que pour mieux s'y inscrire.

Et de se jeter ainsi à corps perdu dans les sillons d'une "identification impossible », ainsi que la décrit Homi Bhabha : tout à la fois renverser le maître, et prendre sa place. Tour assertif que la psyché confère, au moment de sa possible rétroprojection sociale, à la double négation qui signe, avec l'ambivalence du désir du sujet postcolonial, celle de la logique coloniale elle-même : celle d'un sentiment d'identité assis sur le déni de réalité de la perte impulsé par un dispositif de pouvoir social qui voue ainsi ses sujets à refouler tout ce qui menace d'en fragmenter l'unité, pour les contraindre à choisir, comme d'eux-mêmes, de prendre pour unique objet de leur désir la conformité à la norme instituée, et clivant ainsi, par choc en retour, le désir lui-même en bon et mauvais désir. Si Christophe, en effet, agit au nom de la perte elle-même - «On nous vola nos noms »-, c'est pour mieux en rémunérer le défaut « de noms de gloire je veux couvrir vos noms d'esclaves!»-, se précipitant ainsi dans le destin dont il se voit, obstinément, exclure.

Et c'est précisément pour cela qu'il ne saurait y avoir, dans cette tragédie, aucune ironie. Bien plutôt, et au sens fort du terme de mise en dérisoire, une dérision tragique, si mordante soit-elle par endroits, toujours empreinte de fraternelle considération, où celui qui revint d'outre-tombe pour ébranler, coup de tonnerre,

2 Christophe a en effet participé à la révolte victorieuse d'esclaves qui aboutit à l'indépendance de l'ancienne colonie française. Dès 1793, Toussaint Louverture, puis Dessalines, mènent les esclaves insurgés de Saint-Domingue, sous occupation française depuis 1665, à la victoire : le 1er janvier 1804, l'indépendance de la «première République noire » est proclamée, sous le nom retrouvé d'Haïti. Henri Christophe règnera sur la partie Nord d'Haïti, face à son adversaire historique Pétion, au Sud, de 1807 à 1820, date de sa mort. 
l'ordre des vivants, ne put, à vouloir rémunérer le défaut de l'altérité elle-même, que se prendre au piège de son propre et si humain désir d'une perfection faite monde éternité glacée que ne troublerait ni la mort, ni le désir et surtout pas la chair ennemie de sujets aussi récalcitrants à la bataille et à l'ouvrage qu'au mariage.

Fatalement - mais c'est là la loi du réel avant de faire celle de la tragédie qui en suit le cours (il s'agit d'une pièce historique, et pour laquelle Césaire disposait d'une documentation détaillée) -, l'utopie vire en son contraire, et consacre l'échec de celui qui, à vouloir outrepasser non les règles du jeu social, mais bien l'altérité que porte par devers elle l'humaine condition, ne put que se confronter à son tragique et triomphant retour. Christophe est fatalement humain. Ainsi fallait-il que l'utopie se retourne contre son auteur, et que le roi se suicide non seulement pour mettre terme à sa quête mortifère d'idéalité, mais pour rendre la pleine mesure de sa beauté perdue. Car la pièce ne s'arrête pas à la mort de Christophe, mais avec le chant de deuil de ses fidèles et notamment de Madame. Face à l'engouement versatile d'un peuple déjà oublieux d'avoir hier porté aux nues celui dont le corps mort aujourd'hui leur pèse au point de préférer s'en délester en chemin, lui refusant jusqu'à « la cave de boue du scarabée » (151), elle promet quant à elle de toujours porter, jusque dans la vieillesse claudicante, la charge de son nom, et de se faire ainsi la vivante stèle érigée à la mémoire de sa soif vaincue d'idéal.

La Tragédie du roi Christophe est, en effet, célébration sans fin d'un deuil impossible. Célébration de l'idéalité, en tant qu'elle est, tout à fois, irrémédiablement perdue, et irrémédiablement désirée, la pièce, lui offrant son lieu de mémoire, nous offre aussi le lieu social et culturel, jusque là refoulé en-deçà du langage et du sens proprement réduit à l'insensé -, de sa possible appréhension symbolique. Dans l'espace-temps ritualisé de la tragédie, de nous inviter ainsi à faire dans le même mouvement l'acting out, et de la perte, et du désir de la combler : ravivant d'abord l'éclat de son objet fantôme pour le porter ensuite à son point de non retour, elle nous convie à en garder vive la mémoire même, dessinant ainsi une esthétique narrative où la " revenance de l'histoire », selon le titre de l'ouvrage de Jean-François Hamel, n'aurait vœu à résoudre le trouble, ni du passé, ni de l'avenir, mais bien plutôt à troubler la présence elle-même, et à œuvrer ainsi, comme il l'entend dans les toutes dernières lignes, «à ce que le travail de deuil à l'égard du passé [...] ne s'accomplisse pas jusqu'à son terme », et à ce «que les objets perdus conservent leur inquiétante étrangeté » (231).

Car ce qui a été, en dernier comme en premier lieu, perdu, dans ce déni d'altérité en quoi consiste la quête d'identité, et ce si rhizomatique soit-elle, réside bien là, dans cette «trace $e^{3} \mathrm{du}$ "tour" de l'autre sur la scène de l'émergence de l'être » (Butler 286)

${ }^{3} C^{\prime}$ est moi qui souligne. 
qui, pourtant, et si inquiétante soit-elle, seule autorise l'avènement de la socialité. Et c'est précisément de cela, que La tragédie du roi Christophe, œuvre cathartique s'il en est, tient sa force subversive : dans cette "mémoire de l'oubli », ainsi que l'entend Giorgio Agamben, seule susceptible de signifier la perte sans chercher à la résorber et de sauver ainsi, avec le souvenir de l'altérité qui hante l'identification, la possibilité même d'inaugurer une relation à l'autre.

C'est bien en cela, que la Négritude de Césaire me semble, non pas antagoniste, mais bel et bien supplémentaire à la créolisation : dans cette exigence lucide qui porte son auteur à reconnaître, et d'abord en lui-même, l'effet du pouvoir qui l'habite. Car de cette influence, il n'est, sauf utopie, ni possible, ni souhaitable, de s'abstraire - ne serait-ce que dans la mesure où nous ne pouvons nous inscrire à son encontre qu'à la seule condition de s'inscrire aussi en son sein (comme le souligne en effet Gayatri C. Spivak, les subalternes sont, pour leur part, voué-e-s au mutisme). Dans la reconnaissance même, donc, de la volonté d'oubli qui le travaille, et sans laquelle, en fait de relation à l'autre, il n'est possible que d'en reconduire la spécularité - cette stéréotypie, qui est aussi une stéréotopie. Sauver la mémoire de l'oubli de son propre oubli, voilà l'enjeu.

Car à vouloir s'émanciper de « ces marqueurs archaïques que sont la couleur de peau, la langue que l'on parle, le dieu que l'on honore ou celui que l'on craint, le sol où l'on est né » (Chamoiseau et Glissant Les murs 15) pour ne plus valoir que fonction de son imaginaire, qui est aussi volonté d'en libérer son imaginaire pour atteindre « cette plénitude optimale, loin des conquêtes, des aigreurs, des revanches ou des soifs de dominations » (Chamoiseau et Glissant Les murs 15), le risque est bel et bien, pour les tenants de la créolisation, de ne jamais faire que consommer l'illusion de liberté produite par le dispositif de pouvoir identitaire lui-même - elle qui tient, précisément, dans ce déni d'altérité qui ne consacre rien d'autre que la fétichisation de l'image elle-même. Or, quel est, dans un cas comme dans l'autre, cet imaginaire totalement libre, sans entraves, sans frontières, bref, sans limites, sinon la définition même du fantasme?

À la poétique de la Relation, il manque donc quelque chose : la volonté de garder vive cette mémoire de l'oubli, contre la volonté d'oubli elle-même - de sorte qu'aucune plénitude, quand bien même découlerait-elle, non plus d'un, mais bien d'une profusion d'imaginaires, n'ait jamais vœu à se substituer à la perte. La créolisation n'est pas l'alternative de l'aliénation coloniale, pas plus qu'elle ne saurait être celle de la mélancolisation du monde. Au contraire, rien (ne) distingue la négation de l'une de la négativité de l'autre, sinon le refus de l'oubli de cette perte inaugurale, dans le texte matérialisé par les parenthèses, et faute duquel les tenants de la créolisation se vouent, ironiquement, à conforter l'effet de pouvoir du dispositif identitaire là même où ils entendaient s'en affranchir. 
http://ejournals.library.ualberta.ca/index.php/af

Or, à l'heure de la mondialisation, si l'histoire caribéenne peut s'avérer riche d'enseignements, c'est précisément dans ce rappel des dérives utopiques auquel confine, invariablement, le déni de la perte que signe la présence de l'autre - elle qui, pour avoir été encouragée par le dispositif de pouvoir identitaire jusqu'à son point traumatique et colonial, de non retour, fait notre lot commun dès lors que, tout à notre lâcheté retrouvée, nous cédons au désir de nous affranchir de notre propre et inquiétante étrangeté. On ne s'en surprendra pas : en créole, l'usage du réfléchi passe nécessairement par le mot «kò». Aussi bien ne dit-on pas « Je me regarde dans la glace », mais bien « Je regarde mon corps dans la glace » (" Man ka gadé kò-mwen adan glas-la»). Rappel permanent de cette intime altérité qui interdit au «je» de se confondre jamais avec aucune image sociale, mais qui, en en tournant bien plutôt en dérision le désir, l'invite par là même à en relancer le mouvement dans la chair de la socialité. 


\section{Bibliographie}

- Agamben, Giorgio. Ce qui reste d'Auschwitz. Paris : Rivages Poche, 2003 (1 ${ }^{\text {ère édition }}$ 1998).

- Berman, Antoine. L'épreuve de l'étranger. Culture et traduction dans l'Allemagne romantique. Paris : Gallimard, 1984.

- Bhabha, Homi. Les lieux de la culture. Paris : Payot, 2007.

- Butler, Judith. La vie psychique du pouvoir. Paris : Amsterdam, 2002.

- Césaire, Aimé. "Cahier d'un retour au pays natal. » Aimé Césaire. La Poésie. Paris : Seuil, 1994. 7-65.

- Césaire, Aimé. Et les chiens se taisaient. Paris : Présence Africaine, 1974.

- Césaire, Aimé et Khalid Chraibi. "Le Roi Christophe de Haïti : “On nous vola nos noms". » (1965) Oumma.com. 10 mai 2006. 20 Juin 2009 <http://oumma.com/Le-RoiChristophe-de-Haiti-On-nous>

- Césaire, Aimé. La tragédie du roi Christophe. Paris : Présence Africaine, 1963.

- Chamoiseau, Patrick et Edouard Glissant. L'intraitable beauté du monde. Adresse à Barack Obama. Paris : Galaade, 2009.

- Chamoiseau, Patrick et Edouard Glissant. Quand les murs tombent. L'identité nationale hors-la-loi ?. Paris : Galaade, 2007.

- Collectif "Pour une "littérature-monde" en français", Le Monde 16 mars 2007. 8 septembre $2009<$ http://www.lianes.org/Manifeste-pour-une-litterature-monde-enfrancais_a128.html>

- Ehrenberg, Alain. La fatigue d'être soi. Paris : Odile Jacob, 1998.

- Fanon, Frantz. Les damnés de la terre Paris : Poche/La découverte, 2002 (1 ère édition 1961)

- Fanon, Frantz. Peau noire, masques blancs. Paris : Points/Seuil, 1952.

- Glissant, Edouard. Traité du Tout-monde. Poétique IV. Paris : Gallimard, 1997.

- Glissant, Edouard. Poétique de la Relation. Poétique III. Paris : Gallimard, 1990.

- Hamel, Jean-François. Revenances de l'histoire. Répétition, narrativité, modernité. Paris : Editions de Minuit, 2006.

- Lacan, Jacques. «Le stade du miroir comme formateur de la fonction du Je telle qu'elle nous est révélée dans l'expérience psychanalytique. » Ecrits. Paris: Seuil, 1966. 93-100.

- Spivak, Gayatri Chakravorty. Les subalternes peuvent-elles parler?. Paris: Amsterdam, 2009.

- Toumson, Roger. L'utopie perdue des Iles d'Amérique. Paris : Champion, 2004.

- Toumson, Roger. La transgression des couleurs. Littérature et langage dans les Antilles françaises (XIIIe XIXe XXe siècles) I. Paris : Editions Caribéennes, 2000.

- Toumson, Roger. Mythologie du métissage. Paris : PUF, 1998. 